\title{
Characteristics of pre-nuclear pitch accents in statements and yes-no questions in Greek
}

Mary Baltazani

Department of Linguistics, University of Ioannina, Greece

https://doi.org/10.36505/ExLing-2006/01/0014/000014

\begin{abstract}
In this paper I present the results of a production experiment testing the hypothesis that $\mathrm{L}^{*}+\mathrm{H}$ pre-nuclear pitch accents are indistinguishable in statements and ques-tions in Greek. Results show that the $\mathrm{L}$ and the $\mathrm{H}$ tones of the $\mathrm{L}^{*}+\mathrm{H}$ pitch accent have different patterns of alignment in polar and in statements. These results suggest that the pitch accents are realized differently in the two utterance types under inves-tigation. It remains to be explored, through perception experiments, whether the phonetic differences that were found are salient enough for listeners to distinguish between statements and questions. If they are, then the prenuclear pitch accents in statements and those in polar questions belong to different phonological categories.
\end{abstract}

\section{Introduction}

Analyses of the intonation system of Greek maintain that the most frequently used pre-nuclear pitch accent is $\mathrm{L}^{*}+\mathrm{H}$ across all utterance types, statements, questions, negatives, and imperatives (Arvaniti, Ladd and Mennen 1998; Baltazani 2002; Arvaniti and Baltazani 2005).

This state of affairs might give rise to processing problems on the part of the listener for the following reason: In Greek, statements and yes-no questions can be string identical, differing only in the type of nuclear pitch accent and boundary tones, according to the analyses cited above. Therefore, we can make the hypothesis that in utterances with a number of pre-nuclear pitch accents preceding a late nucleus, listeners will have to wait for the nucleus to be uttered before they can determine whether the utterance they are processing is a statement or a question. This hypothesis has not been tested yet, to my knowledge.

\section{Experiment}

To test the hypothesis that pre-nuclear pitch accents are indistinguishable in statements and questions, both production and perception tests were designed. In this paper I present the results of a production experiment.

ExLing 2006: Proceedings of 1st Tutorial and Research Workshop on Experimental Linguistics, 28-30 August 2006, Athens, Greece 


\section{Method}

The same matrix sentence was used for the two utterance types (statement, yes-no question). Each type was preceded by a different context, to elicit the production of the desired melody. The utterances were designed to have three pitch accents, two pre-nuclear ones followed by the nuclear one. Eight speakers participated in this experiment, 3 male and 5 female, producing a corpus of 128 utterances ( 8 speakers X 2 matrix sentences X 2 types X 4 repetitions per speaker).

The tones of the first bi-tonal accent were labelled L1 and H1. The analysis showed a low plateau between the first and the second pitch accent, so two low points were measured for the second pitch accent, L2 and L3, at the two edges of the low plateau. Similarly, there was a high plateau during the second pitch accent, and its two edges were labelled H2 and H3. Figure 1 illustrates the four measuring points of the second pitch accent in a polar question as uttered by one of the female speakers. These points were found in both statements and polar questions.

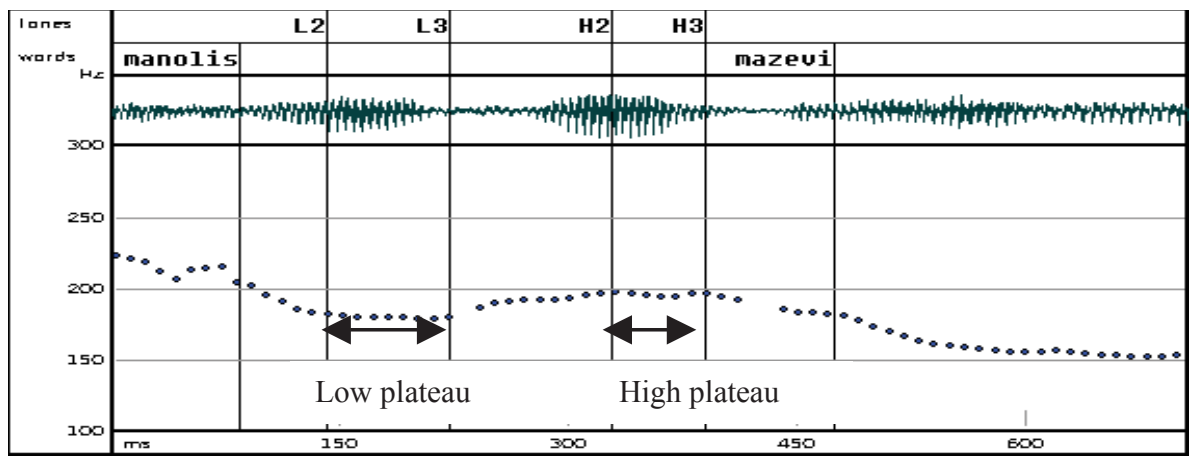

Figure 1. The four measuring points, $\mathrm{L} 2,13, \mathrm{H} 2, \mathrm{H} 3$, of the second $\mathrm{L}^{*}+\mathrm{H}$ pitch accent.

The following measurements were taken. First, F0 of the tones composing the bi-tonal accent for scaling differences in statements and polars. Second, for alignment differences, I took two measurements: a) the distance in time between consecutive tones; $b$ ) the distance in time between tonal targets and segmental landmarks. In particular: b1) L1 to consonant onset of the first stressed syllable; b2) L2 to consonant onset of the second stressed syllable; b3) L3 to consonant onset of the second stressed syllable; b4) H1 to vowel offset of the first stressed syllable; b4) H2 to vowel offset of the second stressed syllable.

No statistical analysis was carried out since the criterion for significance of the results is whether or not pitch accents in the two sentence types are perceived as different by listeners. 


\section{Results}

The realization of the Greek $\mathrm{L}^{*}+\mathrm{H}$ has been decribed as a gradual rise from a trough (the $\mathrm{L}$ tone) to a peak (the $\mathrm{H}$ tone). In general, the $\mathrm{L}$ is aligned at the very beginning or slightly before the onset of the stressed syllable, and the $\mathrm{H}$ early in the first post-stress vowel (Arvaniti, Ladd and Mennen 1998; Baltazani 2002; Arvaniti and Baltazani 2005).

The results of the present experiment show that there is a difference in the realization of the bitonal $\mathrm{L}^{*}+\mathrm{H}$ pitch accent in neutral statements and polar questions. Differences in scaling were not consistent across speakers; however, systematic differences were found in alignment. The scaling differences are presented in the first subsection below, followed by the alignment differences in the second subsection.

\section{Scaling}

Some scaling differences were found, but they depended on gender (Table1). For male speakers, the $\mathrm{L}$ and the $\mathrm{H}$ tones are realized higher in statements than in polars. For female speakers, there is no difference for the L tones across sentence types; the $\mathrm{H}$ tones are realized higher in polars, that is, the reverse trend from what was found for males. No differences were found between statements and polar questions in the pitch range (that is, the difference in $\mathrm{Hz}$ between the $\mathrm{L}$ and the $\mathrm{H}$ ).

Table 1. Average F0 values of $\mathrm{L}$ and $\mathrm{H}$ tones of the $\mathrm{L}^{*}+\mathrm{H}$ pitch accents for male and female speakers in statements and polar questions. Standard deviation is given in parentheses.

\begin{tabular}{|c|c|c|c|c|}
\hline & \multicolumn{2}{|l|}{ L tones } & \multicolumn{2}{|l|}{$\mathrm{H}$ tones } \\
\hline & statement & polar & statement & polar \\
\hline Males & $114(8.6)$ & $105(4.5)$ & $139(11.2)$ & $128(11.7)$ \\
\hline Females & $203(28)$ & 205 (19.1) & $244(34)$ & $254(31.5)$ \\
\hline
\end{tabular}

\footnotetext{
Alignment

In the first $\mathrm{L}^{*}+\mathrm{H}$ pitch accent, no consistent differences were found in the alignment of the $\mathrm{L}$ tone. For 2 of the 8 speakers, the $\mathrm{H}$ tone occurs within the stressed vowel in polar questions. Tones with this alignment are described as $\mathrm{L}+\mathrm{H}^{*}$ in GRToBI (Arvaniti and Baltazani 2005). For the remaining speakers, the $\mathrm{H}$ tone occurs in the post stress syllable and aligns consistently later in polars than in statements (on average $10 \mathrm{~ms}$ difference).

Recall there was a low plateau found after the first pitch accent and that its two edges were labelled L2 and L3. L2 occurred within the pre-stress vowel of the second word in both statements and polars. However, for 6 out of 8 speakers, L2 aligned on average $36 \mathrm{~ms}$ later in neutrals than in polars. The duration of the low plateau (the L2-L3 distance) was on average $30 \mathrm{~ms}$
} 
longer in polars. There were no consistent differences in the alignment of L3 between statements and polars.

Finally, there was a difference found in the alignment of the two $\mathrm{H}$ tones at the edges of the high plateau. $\mathrm{H} 2$ occurred within the post-stress consonant in both sentence types, but it aligned on average $40 \mathrm{~ms}$ earlier in neutrals than in polars. No other consistent differences were found.

\section{Discussion and conclusion}

The results suggest that the pitch accents are realized differently in the two utterance types under investigation. The low plateau that was found between the two pitch accents is longer in polars than in statements: Its left edge occurs earlier in polars than in statements and its right edge occurs later. Moreover, the high peak of the second pitch accent occurs later in statements. In geometrical terms, polar questions show a longer low plateau which has more abrupt slopes at its two edges. However, it remains to be explored, through perception experiments, whether these phonetic differences are salient enough and whether listeners actually use them to distinguish between statements and questions.

The results of this production experiment, in combination with the results of follow up perception experiments have theoretical implications. If the phonetic differences detected here turn out to be salient enough to help listeners distinguish between statements and questions, then they have the status of phonological categories. In other words, intonational theory will have to expand the inventory of pitch accents to include the type found in polar questions. If, on the other hand, listeners cannot distinguish between the prenuclear pitch accents in statements from those in polar questions, then the different realizations of the $\mathrm{L}^{*}+\mathrm{H}$ found must be merely phonetic 'allotones' of the same tonal category.

\section{References}

Arvaniti, A. 2002. The intonation of yes-no questions in Greek. In M. MakriTsilipakou (ed.), Selected Papers on Theoretical and Applied Linguistics, 71-83. Thessaloniki: Department of Theoretical and Applied Linguistics, School of English, Aristotle University.

Arvaniti, A., Ladd, R.D., and Mennen, I. 1998. Stability of Tonal Alignment: The Case of Greek Prenuclear Accents, Journal of Phonetics, 26: 3-25.

Arvaniti, A., and Baltazani, M. 2005. Intonational Analysis and Prosodic Annotation of Greek Spoken Corpora. In Jun, S.A (ed.), Prosodic Typology and Transcription. Oxford University Press.

Baltazani, M., 2002. Quantifier scope and the role of intonation in Greek. PhD Thesis, UCLA. 